1 Hacettepe Journal of Mathematics and Statistics

$\bigcap$ Volume 45 (5) (2016), 1355-1366

\title{
Weakly second modules over noncommutative rings
}

\author{
R. Beyranvand ${ }^{* \dagger}$ and F. Rastgoo
}

\begin{abstract}
Let $R$ be an arbitrary ring. In this paper we will introduce the concept of a weakly second $R$-module (a generalization of the second $R$-module) and we will obtain some related results.
\end{abstract}

Keywords: Weakly second module; second module; prime module; weakly prime module.

2000 AMS Classification: 16D10; 16D90; 16N60

Received : 18.08.2015 Accepted : 06.11.2015 Doi : 10.15672/HJMS.20164515691

\section{Introduction}

Throughout this paper, all rings will have identity elements and all modules will be right unitary. We use the notation " $\subset$ " to denote strict inclusion. Unless otherwise stated, $R$ denotes an arbitrary ring with identity element. Let $M$ be an $R$-module. Then the annihilator of $M$ (in $R$ ) is the ideal $\operatorname{ann}_{R}(M)=\{r \in R \mid M r=0\}$. Also for any submodule $N$ of $M$ and any ideal $I$ of $R$, the submodule $\{x \in M \mid x I \subseteq N\}$ of $M$ is denoted by $(N: M I)$.

Recall that a nonzero $R$-module $M$ is prime if $\operatorname{ann}_{R}(M)=\operatorname{ann}_{R}(N)$ for every nonzero submodule $N$ of $M$. Also a nonzero $R$-module $M$ is called weakly prime in case $\operatorname{ann}_{R}(N)$ is a prime ideal of $R$ for every nonzero submodule $N$ of $M$. By a (weakly) prime submodule of a module $M$ we mean a submodule $N$ such that the module $M / N$ is (weakly) prime. The notion of prime modules first was introduced by Dauns in [11]. Also in [9], Behboodi and Koohi introduced the notion of weakly prime modules and investigated the properties of this class of modules. More details about prime modules and weakly prime modules can be found in [2, 5, 6, 9].

On the other hand, a nonzero module $M$ is called a second module (the dual notion of a prime module) provided $\operatorname{ann}_{R}(M)=\operatorname{ann}_{R}(M / N)$ for every proper submodule $N$ of $M$. This notion was introduced by Yassemi in [15], for modules over commutative rings.

*Department of Mathematics, Lorestan University, Khorramabad, Iran

Email: beyranvand.r@lu.ac.ir

$\dagger$ Corresponding Author.

‡Department of Mathematics, Lorestan University, Khorramabad, Iran, Email: rastgoo_fahimeh@yahoo.com 
Moreover, in [10], the authors generalized second modules from commutative rings to noncommutative setting.

The purpose of this paper is to introduce and study the concept of weakly second modules (the dual of weakly prime modules) over noncommutative rings. A nonzero $R$-module $M$ is called a weakly second module if $\operatorname{ann}_{R}(M / N)$ is a prime ideal of $R$ for every proper submodule $N$ of $M$. It is clear that every second module is a weakly second module. By a (weakly) second submodule of a module we mean a submodule which is also a (weakly) second module. In addition to obtaining some useful information about this class of modules, we investigate which dual of the given results about the weakly prime modules hold for the weakly second ones. For a right $R$-module $M$, among other results, we prove the following statements:

- Theorem 2.3. ((1), (2), (8)) $M$ is a weakly second module if and only if for every two ideals $I$ and $J$ of $R, M I J=M I$ or $M I J=M J$, if and only if the set $\left\{\operatorname{ann}_{R}(M / N) \mid N\right.$ is a proper submodule of $M\}$ is a chain of prime ideals of $R$.

- Theorem 2.5 and Theorem 2.6. Secondness and weakly secondness are Morita invariant properties.

- Proposition 2.7. $M$ is weakly second if and only if for every proper submodule $K$ of $M$, there is a prime ideal $I$ of $R$ contained in $\operatorname{ann}_{R}(M / K)$ such that $M / K$ cogenerates $R / I$.

- Corollary 2.9. If $R$ is a right Artinian ring, then $M$ is a weakly second $R$-module if and only if $M$ is a homogenous semisimple $R$-module.

- Theorem 2.15. If $M$ satisfies the descending chain condition on weakly socle submodules, then every nonzero submodule of $M$ has only a finite number of maximal weakly second submodule.

- Proposition 2.17. If $R$ satisfies the ascending chain condition on prime ideals, then $M$ has a second submodule if and only if it has a weakly second submodule.

- Theorem 3.5. Let $R$ be a ring whose two-sided ideals satisfy ACC. Then a nonzero $R$-module $M$ is weakly second if and only if for every two prime ideals $I$ and $J$ of $R$, $M I J=M I$ or $M I J=M J$.

- Proposition 3.7. Let $R$ be a prime right Goldie ring. Then every nonzero properly divisible $R$-module is a weakly second module.

\section{Weakly second modules}

We begin this section with the definition of weakly second modules and then some remarks and examples are given.

2.1. Definition. A nonzero right $R$-module $M$ is called weakly second if for every proper submodule $N$ of $M, \operatorname{ann}_{R}(M / N)$ is a prime ideal of $R$.

2.2. Examples. (a) The $\mathbb{Z}$-module $\mathbb{Z}_{n}$ is a weakly second if and only if $n$ is a prime number.

(b) If $n \neq m$, then the $\mathbb{Z}$-module $\mathbb{Z}_{n} \oplus \mathbb{Z}_{m}$ is not weakly second.

(c) Let $D$ be a division ring and $V=\oplus_{i=1}^{\infty} e_{i} D$ be a vector space over $D$. Set $R=$ $\operatorname{End}\left(V_{D}\right)$ and $T=\{f \in R \mid \operatorname{rank} f<\infty\}$. It is known that $R$ has only three ideals $(0)$, $R$ and $T$. So $T$ is a maximal ideal and (0) is a prime ideal of $R$. Now it is easy to check that $R$ as a left $R$-module is weakly second but is not a second $R$-module. 
(d) Let $p$ and $q$ be two distinct prime numbers. Consider the $\mathbb{Z}$-modules

$$
M=<1 / p+\mathbb{Z}>\oplus<1 / q+\mathbb{Z}>\oplus \mathbb{Z}_{p} \infty,
$$

and

$$
N=<1 / p+\mathbb{Z}>\oplus(0) \oplus \mathbb{Z}_{p^{\infty}} .
$$

It is easily checked that $N$ and $M / N$ are weakly second modules, but $M$ is not a weakly second module.

(e) Recall that a submodule $N$ of an $R$-module $M$ is fully invariant if for every $R$ endomorphism $f: M \rightarrow M, f(N) \subseteq N$. A right $R$-module $M$ is a weakly second module if and only if for every fully invariant proper submodule $N$ of $M, \operatorname{ann}_{R}(M / N)$ is a prime ideal of $R$. To see this, let $I$ and $J$ be two ideals of $R$ and $L$ be a proper submodule of $M$ such that $I J \subseteq \operatorname{ann}_{R}(M / L)$. Since $M I J$ is a fully invariant proper submodule of $M, \operatorname{ann}_{R}(M / M I J)$ is a prime ideal of $R$. Now $I J \subseteq \operatorname{ann}_{R}(M / M I J)$ implies that $M I \subseteq M I J \subseteq L$ or $M J \subseteq M I J \subseteq L$, and so $I \subseteq \operatorname{ann}_{R}(M / L)$ or $J \subseteq \operatorname{ann}_{R}(M / L)$. Thus $\operatorname{ann}_{R}(M / L)$ is prime.

In the following theorem, some characterizations of weakly second modules are given.

2.3. Theorem. For a nonzero right $R$-module $M$, the following statements are equivalent:

(1) $M$ is a weakly second module;

(2) For every two ideals $I$ and $J$ of $R, M I J=M I$ or $M I J=M J$;

(3) For every two ideals $I$ and $J$ of $R, J \nsubseteq$ ann $_{R}(M / M I J)$ implies that $M I J=M I$;

(4) For every two ideals $I$ and $J$ of $R, I \nsubseteq a_{n} n_{R}(M / M I J)$ implies that $M I J=M J$;

(5) For every two ideals $I$ and $J$ of $R$, ann $_{R}(M / M I J) \subset I$ implies that $M I J=M J$;

(6) For every two ideals $I$ and $J$ of $R$, ann $n_{R}(M / M I J) \subset J$ implies that $M I J=M I$;

(7) Every nonzero quotient of $M$ is weakly second;

(8) The set $\left\{\operatorname{ann}_{R}(M / N) \mid N\right.$ is a proper submodule of $\left.M\right\}$ is a chain of prime ideals of $R$.

Proof. (1) $\Rightarrow(2)$. Suppose that $M$ is weakly second and $I$ and $J$ are two ideals of $R$. If $M I J=M$, then $M I J=M I$ and $M I J=M J$. So suppose that $M I J$ is a proper submodule of $M$ and hence $\operatorname{ann}_{R}(M / M I J)$ is a prime ideal of $R$. Since $I J \subseteq$ $\operatorname{ann}_{R}(M / M I J), I \subseteq \operatorname{ann}_{R}(M / M I J)$ or $J \subseteq \operatorname{ann}_{R}(M / M I J)$. Thus $M I J=M I$ or $M I J=M J$.

$(2) \Rightarrow(1)$. Let $N$ be a proper submodule of $M$ and $I J \subseteq \operatorname{ann}_{R}(M / N)$ for some two ideals $I$ and $J$ of $R$. Then $M I J \subseteq N$ and by the hypothesis $M I \subseteq N$ or $M J \subseteq N$. Thus $\operatorname{ann}_{R}(M / N)$ is prime.

$(2) \Leftrightarrow(3)$ and $(2) \Leftrightarrow(4)$ are clear.

$(4) \Rightarrow(5)$. Let $I$ and $J$ be two ideals of $R$ such that $\operatorname{ann}_{R}(M / M I J) \subset I$. Then $I \nsubseteq$ $\operatorname{ann}_{R}(M / M I J)$ and so $M I J=M J$.

$(5) \Rightarrow(4)$. Suppose that $I$ and $J$ are two ideals of $R$ and $I \nsubseteq \operatorname{ann}_{R}(M / M I J)$. Then $\operatorname{ann}_{R}(M / M I J) \subset I+\operatorname{ann}_{R}(M / M I J)$ and we have $\operatorname{ann}_{R}(M / M I J)=\operatorname{ann}_{R}(M / M(I+$ $\left.\left.\operatorname{ann}_{R}(M / M I J)\right) J\right)$. Thus $\operatorname{ann}_{R}\left(M / M\left(I+\operatorname{ann}_{R}(M / M I J)\right) J\right) \subset I+\operatorname{ann}_{R}(M / M I J)$ and by (5), $M\left(I+\operatorname{ann}_{R}(M / M I J)\right) J=M J$. But $M\left(I+\operatorname{ann}_{R}(M / M I J)\right) J \subseteq M I J$ and so $M I J=M J$.

$(3) \Rightarrow(6)$ is similar to $(4) \Rightarrow(5)$ and $(6) \Rightarrow(3)$ is similar to $(5) \Rightarrow(4)$.

$(1) \Rightarrow(7)$ is clear. 
$(7) \Rightarrow(8)$. Let $N_{1}$ and $N_{2}$ be two proper submodules of $M, P=\operatorname{ann}_{R}\left(M / N_{1}\right)$ and $Q=$ $\operatorname{ann}_{R}\left(M / N_{2}\right)$. If $P \nsubseteq Q$ and $Q \nsubseteq P$, then there exist two ideals $I_{1}$ and $I_{2}$ of $R$ such that $I_{1} \subseteq P, I_{2} \subseteq Q, I_{1} \nsubseteq Q$ and $I_{2} \nsubseteq \subseteq$. Since $\operatorname{ann}_{R}\left(M /\left(N_{1} \cap N_{2}\right)\right)$ is a prime ideal of $R$ and $I_{1} I_{2} \subseteq \operatorname{ann}_{R}\left(M /\left(N_{1} \cap N_{2}\right)\right), I_{1} \subseteq \operatorname{ann}_{R}\left(M /\left(N_{1} \cap N_{2}\right)\right)$ or $I_{2} \subseteq \operatorname{ann}_{R}\left(M /\left(N_{1} \cap N_{2}\right)\right)$. But $\operatorname{ann}_{R}\left(M /\left(N_{1} \cap N_{2}\right)\right) \subseteq \operatorname{ann}_{R}\left(M / N_{2}\right)=Q$ and $\operatorname{ann}_{R}\left(M /\left(N_{1} \cap N_{2}\right)\right) \subseteq \operatorname{ann}_{R}\left(M / N_{1}\right)=P$. Thus $I_{1} \subseteq Q$ or $I_{2} \subseteq P$, a contradiction.

$(8) \Rightarrow(1)$. Clear.

Next, we show that both secondness and weakly secondness are Morita invariant properties.

2.4. Theorem. Secondness is a Morita invariant property.

Proof. Let $R$ and $S$ be Morita equivalent rings via an equivalence $F: \operatorname{Mod}_{R} \rightarrow \operatorname{Mod}_{S}$. Suppose that $M$ is a second $R$-module. Let $I=\operatorname{ann}_{R}(M)$ and $B=\operatorname{ann}_{S}(F(R / I))$. By [1, Proposition 21.11], $R / I$ is Morita equivalent to $S / B$. Also by [1, Proposition 21.6], $F(M)$ is faithful as an $S / B$-module because $M$ is a faithful $R / I$-module. Thus $B=$ $\operatorname{ann}_{S}(F(M))$. Now assume that $N$ is a proper $S$-submodule of $F(M)$. We show that $\operatorname{ann}_{S}(F(M))=\operatorname{ann}_{S}(F(M) / N)$. For a submodule $K$ of $M$, let $i_{K \leq M}: K \rightarrow M$ denote the inclusion monomorphism. Since by [1, Proposition 21.7], the mapping defined by $\Lambda_{M}: K \rightarrow \operatorname{Im} F\left(i_{K \leq M}\right)$ is a lattice isomorphism from the lattice of submodules of $M$ onto the lattice of submodules of $F(M)$, there exists $K \leq M$ such that $\Lambda_{M}(K)=$ $N=\operatorname{Im} F\left(i_{K \leq M}\right)$. Since Morita equivalences preserve exactness, $F(M) / \operatorname{Im} F\left(i_{K \leq M} \cong\right.$ $F(M / K)$. Therefore $F(M) / N \cong F(M / K)$ and so $\operatorname{ann}_{S}(F(M) / N)=\operatorname{ann}_{S}(F(M / K))$. On the other hand, since $M$ is a second $R$-module, $I=\operatorname{ann}_{R}(M)=\operatorname{ann}_{R}(M / K)$ and so by the first part of the proof, $B=\operatorname{ann}_{S}(F(M))=\operatorname{ann}_{S}(F(M / K))$. Thus $\operatorname{ann}_{S}(F(M))=$ $\operatorname{ann}_{S}(F(M) / N)$ and this implies that $F(M)$ is a second $S$-module, as desired.

2.5. Theorem. Weakly secondness is a Morita invariant property.

Proof. Let $R$ and $S$ be Morita equivalent rings via an equivalence $F: \operatorname{Mod}_{R} \rightarrow \operatorname{Mod}_{S}$. Suppose that $M$ is a weakly second $R$-module and $N$ is a proper $S$-submodule of $F(M)$. We show that $\operatorname{ann}_{S}(F(M) / N)$ is a prime ideal of $S$. In the notations of the proof of above theorem, there exists $K \leq M$ such that $\Lambda_{M}(K)=N=\operatorname{Im} F\left(i_{K \leq M}\right)$ and $\operatorname{ann}_{S}(F(M) / N)=\operatorname{ann}_{S}(F(M / K))$. Let $I=\operatorname{ann}_{R}(M / K)$. Again by the proof of above theorem, $R / I$ is Morita equivalent to $S / B$, where $B=\operatorname{ann}_{S}(F(R / I))=\operatorname{ann}_{S}(F(M / K))$. Since $M$ is a weakly second $R$-module, $I=\operatorname{ann}_{R}(M / K)$ is a prime ideal of $R$ and hence $R / I$ is a prime ring. By [13, Corollary 18.45], $S / B$ is also a prime ring. Thus $B=$ $\operatorname{ann}_{S}(F(M / K))=\operatorname{ann}_{S}(F(M) / N)$ is a prime ideal of $S$, as desired.

2.6. Remark. For a nonzero $R$-module $M$, we note that $M$ is a second $R$-module if and only if for any proper submodule $K$ of $M, M / K$ cogenerates $R / \operatorname{ann}_{R}(M)$. To see this, suppose that $M$ is second and $K$ is a proper submodule of $M$. Then $r+$ $\operatorname{ann}_{R}(M) \rightarrow(x r+K)_{x \in M}$ is an $R$-monomorphism of $R / \operatorname{ann}_{R}(M)$ into $\prod_{x \in M} M / K$. Thus $R / \operatorname{ann}_{R}(M)$ is cogenerated by $M / K$. For the other direction, assume that $f$ : $R / \operatorname{ann}_{R}(M) \rightarrow \prod_{\alpha \in A} M / K$ is an $R$-monomorphism, where $K$ is a proper submodule of $M$. Let $f(\overline{1})=\left(x_{\alpha}+K\right)_{\alpha \in A}$. If $r \in \operatorname{ann}_{R}(M / K)$, then $f(\bar{r})=\left(x_{\alpha} r+K\right)_{\alpha \in A}=0$ and so $\bar{r}=0$. Thus $r \in \operatorname{ann}_{R}(M)$. This yields that $\operatorname{ann}_{R}(M)=\operatorname{ann}_{R}(M / K)$ and hence $M$ is a second module.

Now for weakly second modules, we have the following statement.

2.7. Proposition. Let $M$ be a nonzero $R$-module. Then $M$ is weakly second if and only if for every proper submodule $K$ of $M$, there is a prime ideal $I$ of $R$ contained in $\operatorname{ann}_{R}(M / K)$ such that $M / K$ cogenerates $R / I$. 
Proof. First suppose that $M$ is a weakly second module and $K$ is a proper submodule of $M$. Then $\operatorname{ann}_{R}(M / K)$ is a prime ideal and clearly $M / K \operatorname{cogenerates} R / \operatorname{ann}_{R}(M / K)$. Conversely, suppose that $K$ is a proper submodule of $M$ and $I$ is a prime ideal of $R$ contained in $\operatorname{ann}_{R}(M / K)$ such that $R / I$ is cogenerated by $M / K$. Say $f: R / I \rightarrow \prod_{\alpha \in A} M / K$ is an $R$-monomorphism. Then $\operatorname{ann}_{R}(M / K)=\operatorname{ann}_{R}\left(\prod_{\alpha \in A} M / K\right) \subseteq \operatorname{ann}_{R}(R / I) \subseteq I$ and hence $I=\operatorname{ann}_{R}(M / K)$.

Recall that a module $M$ is homogeneous semisimple if $M$ is a direct sum of pairwise isomorphic simple submodules. Clearly, any homogeneous semisimple module is (weakly) second. We show that the converse is true when $R$ is an Artinian ring. First the following lemma is needed.

2.8. Lemma. Let $R$ be a ring in which every prime ideal is maximal. For a nonzero $R$-module $M$, consider the following statements:

(1) $M$ is prime;

(2) $M$ is weakly prime;

(3) $M$ is second;

(4) $M$ is weakly second;

(5) $M$ is homogeneous semisimple.

Then $(1) \Leftrightarrow(2) \Leftrightarrow(3) \Leftrightarrow(4) \Leftarrow(5)$. Moreover, if in addition $R$ is a commutative ring, then all five statements are equivalent.

Proof. (1) $\Rightarrow(2),(3) \Rightarrow(4)$ and (5) $\Rightarrow(4)$ are trivial.

$(2) \Rightarrow(3)$. Suppose that $M$ is weakly prime. Then $\operatorname{ann}_{R}(M)$ is a prime ideal and hence is a maximal ideal. Thus for any proper submodule $N$ of $M, \operatorname{ann}_{R}(M) \subseteq \operatorname{ann}_{R}(M / N) \subset R$ implies that $\operatorname{ann}_{R}(M)=\operatorname{ann}_{R}(M / N)$ and so $M$ is a second module.

$(4) \Rightarrow(1)$. Suppose $M$ is a weakly second module. Then $\operatorname{ann}_{R}(M)$ is a prime ideal and hence is a maximal ideal. Thus $\operatorname{ann}_{R}(M) \subseteq \operatorname{ann}_{R}(N) \subset R$ implies that $\operatorname{ann}_{R}(M)=$ $\operatorname{ann}_{R}(N)$, for every nonzero submodule $N$ of $M$. It follows that $M$ is a prime module. $(4) \Rightarrow(5)$. Suppose that $R$ is a commutative $\operatorname{ring}$ and $M$ is a weakly second $R$-module. Then $\operatorname{ann}_{R}(M)$ is a prime ideal and by the hypothesis, it is a maximal ideal. Thus $R / P$ is a field where $P=\operatorname{ann}_{R}(M)$. This implies that $M$ is a homogeneous semisimple as $R / P$-module and as $R$-module.

2.9. Corollary. Let $R$ be a right Artinian ring. Then for any nonzero right $R$-module $M$, the five statements in the previous lemma are all equivalent.

Proof. We only prove that if $M$ is a weakly second $R$-module, then it is a homogeneous semisimple $R$-module. Since $M$ is weakly second, $\operatorname{ann}_{R}(M)$ is a prime ideal and so $R / P$ is a right Artinian prime ring where $P=\operatorname{ann}_{R}(M)$. By the Wedderburn-Artin Theorem [12, Theorem 3.5], we conclude that $M$ is a homogeneous semisimple as $R / P$-module and as $R$-module.

2.10. Corollary. Let $R$ be a commutative von Neumann regular ring and $M$ be an $R$ module. Then the following statements are equivalent:

(1) $M$ is second;

(2) $M$ is weakly second;

(3) $M$ is homogeneous semisimple.

Proof. It is well known that every prime ideal in a commutative von Neumann regular ring is a maximal ideal. Now apply Lemma 2.8 . 
The next two results were proved for second modules. See [10, Corollary 2.4 and Proposition 3.6].

2.11. Corollary. Let $A$ be an ideal of a ring $R$ and $M$ be a nonzero right $R$-module such that $M A=0$. Then the $R$-module $M$ is a weakly second module if and only if the $R / A$-module $M$ is a weakly second module.

Proof. Suppose first that the $R$-module $M$ is weakly second and let $I$ and $J$ be two ideals of $R$ containing $A$. Then $M(I / A)(J / A)=M(I J+A / A)=M I J$ and so by Theorem 2.3, $M(I / A)(J / A)=M(I / A)$ or $M(I / A)(J / A)=M(J / A)$. Conversely, suppose that the $R / A$-module $M$ is a weakly second module. For any two ideals $I$ and $J$ of $R$, $M I J=M(I J+A)=M((I J+A) / A)=M((I+A) / A)((J+A) / A)$. Using Theorem 2.3, we have $M I J=M((I+A) / A)=M I$ or $M I J=M((J+A) / A)=M J$, as desired.

Let $R$ be a ring. An ideal $A$ of $R$ is called right T-nilpotent if for any sequence $\left\{a_{1}, a_{2}, \ldots\right\}$ in $A$, there exists a positive integer $n$ such that $a_{n} \ldots a_{1}=0$.

2.12. Proposition. Let $A$ be a right $T$-nilpotent ideal of a ring $R$ and $\bar{R}=R / A$. Then every nonzero right $R$-module $M$ has a proper submodule $N$ such that $M / N$ is a weakly second $R$-module if and only if, every nonzero right $\bar{R}$-module $M$ has a proper submodule $N$ such that $M / N$ is a weakly second $\bar{R}$-module.

Proof. Suppose first that every nonzero right $R$-module $M$ has a proper submodule $N$ such that $M / N$ is a weakly second $R$-module. Let $K$ be a nonzero right $\bar{R}$-module. Then $K$ is a right $R$-module and by the hypothesis, there exists a proper submodule $L$ of $K$ such that the $R$-module $K / L$ is weakly second. It follows that the $\bar{R}$-module $K / L$ is a weakly second module. Conversely, suppose that every nonzero right $\bar{R}$-module $M$ has a proper submodule $N$ such that $M / N$ is a weakly second $\bar{R}$-module. Let $X$ be a nonzero right $R$-module. Then by [1, Lemma 28.3], $X \neq X A$ and so $X /(X A)$ is a nonzero right $\bar{R}$-module. Also by the hypothesis, there exists a proper submodule $Y$ of $X$ containing $X A$, such that the $\bar{R}$-module $X / Y$ is weakly second. Now by Corollary $2.11, X / Y$ is a weakly second $R$-module.

In [10, Proposition 4.2], it is shown that the union of a chain of second submodules of a module is also second. Here, we show that a similar result holds for a directed set of weakly second submodules of a module.

2.13. Lemma. Let $R$ be a ring, and let $N_{i}(i \in I)$ be a directed set of weakly second submodules of a right $R$-module $M$. Then $N=\bigcup_{i \in I} N_{i}$ is a weakly second $R$-module.

Proof. Note that $N$ is a nonzero submodule of $M$. Let $A$ and $B$ be two ideals of $R$. By Theorem 2.3, it suffices to show that $N A B=N A$ or $N A B=N B$. If there exists $k \in I$ such that for each $i \in I, N_{j}=\sup \left\{N_{i}, N_{k}\right\}$ satisfies $N_{j} A B=N_{j} A$, then for each $i \in I$, we have $N_{i} A \subseteq N_{j} A=N_{j} A B \subseteq N A B$. Thus $N A=N A B$. Now suppose that for every $k \in I$, there exists $i \in I$ such that $N_{j}=\sup \left\{N_{i}, N_{k}\right\}$ dose not satisfy $N_{j} A B=N_{j} A$. Since $N_{j}$ is weakly second, $N_{j} B=N_{j} A B$. Then for each $i \in I$, we have $N_{i} B \subseteq N_{j} B=N_{j} A B \subseteq N A B$. Thus $N B=N A B$.

By a maximal weakly second submodule of a module $M$, we mean a weakly second submodule $L$ of $M$ such that $L$ is not properly contained in another weakly second submodule of $M$.

2.14. Corollary. Let $M$ be any nonzero module. Then every weakly second submodule of $M$ contained in a maximal weakly second submodule.

Proof. By Lemma 2.13 and Zorn's Lemma. 
Let $N$ be a submodule of a right $R$-module $M$. We define the weakly socle of $N$ as the sum of all weakly second submodules of $M$ contained in $N$, denoted by $W \cdot \operatorname{soc}(N)$. The weakly socle of $N$ is defined to be $(0)$ in case $N$ dose not contain any weakly second submodule. $N$ is said to be a weakly socle submodule of $M$ if $N \neq 0$ and $W \cdot \operatorname{soc}(N)=N$.

2.15. Theorem. Let $M$ be a right $R$-module. If $M$ satisfies the descending chain condition on weakly socle submodules, then every nonzero submodule of $M$ has only a finite number of maximal weakly second submodule.

Proof. Suppose that the result is false. Then there exists a nonzero submodule $N$ of $M$ such that it has an infinite number of maximal weakly second submodules. Thus $W \cdot \operatorname{soc}(N)$ is a weakly socle submodule of $M$ and $W \cdot \operatorname{soc}(N)$ has an infinite number of maximal weakly second submodules. By the assumption, let $S$ be a weakly socle submodule of $M$ chosen minimal such that $S$ has an infinite number of maximal weakly second submodules. If $S$ is weakly second, then every maximal weakly second submodule contained in $S$ is equal to $S$. Thus $S$ has not an infinite number of maximal weakly second submodules, a contradiction. Therefore $S$ is not weakly second and so there exist two ideals $I$ and $J$ of $R$ and a proper submodule $K$ of $M$ such that $S I J \subseteq K, S I \nsubseteq K$ and $S J \nsubseteq K$. Thus $S \nsubseteq(K: S I)$ and $S \nsubseteq(K: S J)$. Therefore $S \nsubseteq W \cdot \operatorname{soc}((K: S I))$ and $S \nsubseteq W \cdot \operatorname{soc}((K: S J))$. Now we conclude that $W \cdot \operatorname{soc}\left(\left(K:_{S} I\right)\right) \subset S$ and $W \cdot \operatorname{soc}((K: S I)) \subset S$. Let $V$ be a maximal weakly second submodules of $M$ contained in $S$. Then $V I J \subseteq S I J \subseteq K$ and hence $V I \subseteq K$ or $V J \subseteq K$. Thus $V \subseteq\left(K:_{S} I\right)$ or $V \subseteq\left(K:_{S} J\right)$ so that $V \subseteq W \cdot \operatorname{soc}\left(\left(K:_{S} I\right)\right)$ or $V \subseteq W \cdot \operatorname{soc}\left(\left(K:_{S} J\right)\right)$. The minimality of $S$, implies that both $W \cdot \operatorname{soc}\left(\left(K:_{S} I\right)\right)$ and $W \cdot \operatorname{soc}\left(\left(K:_{S} J\right)\right)$ have only finitely many maximal weakly second submodules. Therefore there is only a finite number of possibilities for the module $S$, which is a contradiction.

The following result is immediately obtained.

2.16. Corollary. Every nonzero Artinian module contains only a finite number of maximal weakly second submodule.

Clearly, if an $R$-module has a second submodule, then it has a weakly second submodule. Now, we show that the converse is true when a certain set of ideals of $R$ has the descending chain condition (briefly, DCC). In fact, it is the dual statement of [9, Proposition 5.1].

2.17. Proposition. Let $R$ be a ring whose prime ideals satisfy $D C C$ and let $M$ be a right $R$-module. Then $M$ has a second submodule if and only if it has a weakly second submodule.

Proof. Suppose that $N$ is a weakly second submodule of $M$. Let $I=\operatorname{ann}_{R}(N)$. Since $I$ is a prime ideal, $R / I$ is a prime ring and so $N$ is a faithful $R / I$-module. Without loss of generality, we may assume that $R$ is a prime ring and $M$ is a faithful weakly second module. By Theorem 2.3, the set $T=\left\{\operatorname{ann}_{R}(M / K) \mid K\right.$ is a proper submodule of $\left.M\right\}$ is a chain of prime ideals. If $T=\{0\}$, then $M$ is a second module and we are through. Thus suppose that the chain $T$ contains a nonzero element. Let $L_{0}=\bigcap\{L \subset M \mid 0 \neq$ $\left.\operatorname{ann}_{R}(M / L) \in T\right\}$. Clearly, $L_{0}$ is a submodule of $M$. By the hypothesis, assume that $P$ is a minimal among nonzero elements of $T$. Then $P=\operatorname{ann}_{R}(M / K)$ for some proper submodule $K$ of $M$. We claim that $P=\operatorname{ann}_{R}\left(M / L_{0}\right)$. Since $T$ is a chain, for any proper submodule $L$ of $M$ with $\operatorname{ann}_{R}(M / L) \neq 0$, we have $\operatorname{ann}_{R}(M / L) \subseteq \operatorname{ann}_{R}(M / K)$ or $\operatorname{ann}_{R}(M / K) \subseteq \operatorname{ann}_{R}(M / L)$. The minimality of $P$ implies that $P \subseteq \operatorname{ann}_{R}(M / L)$. Thus $M P \subseteq L$ for any proper submodule $L$ of $M$ with $\operatorname{ann}_{R}(M / L) \neq 0$. It follows that $M P \subseteq L_{0}$ and hence $P \subseteq \operatorname{ann}_{R}\left(M / L_{0}\right)$. By the definition of $L_{0}$, we have $P=$ 
$\operatorname{ann}_{R}\left(M / L_{0}\right)$. Now we show that $L_{0}$ is a second submodule of $M$. Suppose that $I$ is an ideal of $R$ and $L_{0} I \neq 0$. Since $M P \subseteq L_{0}, M P I \subseteq L_{0} I$. But $R$ is a prime ring and so $P I \neq 0$. Thus $\operatorname{ann}_{R}\left(M / L_{0} I\right) \neq 0$ and by the definition of $L_{0}$, we conclude that $L_{0} \subseteq L_{0} I$ and hence $L_{0}=L_{0} I$, as desired.

\section{Further results related to weakly second modules}

In this section, we start with some definitions. Then the relationships between weakly second, weakly prime and second modules are investigated. Let $R$ be a ring and $M$ be a right $R$-module. Then:

(i) $M$ is called a multiplication module if for every submodule $N$ of $M$ there exists an ideal $I$ of $R$ such that $N=M I$. This notion was introduced by Baranard in [7].

(ii) $M$ is called a comultiplication module if for every submodule $N$ of $M$ there exists an ideal $I$ of $R$ such that $\left(0:_{M} I\right)=N$. This notion is introduced by Ansari-Toroghy and Farshadifar in [3].

(iii) A submodule $N$ of $M$ is called secondary submodule if for every ideal $I$ of $R, N I=N$ or there exists a positive integer $n$ such that $N I^{n}=0$.

3.1. Theorem. Let $R$ be a ring and $M$ be a nonzero right $R$-module. Then:

(1) If $M$ is a multiplication module such that ann $n_{R}(M)$ is a prime ideal, then it is prime;

(2) If $M$ is a comultiplication module such that $a n_{R}(M)$ is a prime ideal, then it is second;

(3) A submodule $N$ of $M$ is second if and only if it is both a weakly second and a secondary submodule of $M$;

(4) If any two prime ideals of $R$ are comparable, i.e., $I \subseteq J$ or $J \subseteq I$ for every two prime ideals $I$ and $J$ of $R$, then any sum of weakly second submodules of $M$ is a weakly second submodule of $M$.

Proof. (1) Suppose that $N$ is a nonzero submodule of $M$ and $N I=0$, where $I$ is an ideal of $R$. Since $M$ is multiplication, $N=M J$ for some ideal $J$ of $R$. Then $N I=M J I=0$ and so $M I=0$ because $\operatorname{ann}_{R}(M)$ is prime. Thus $M$ is a prime module.

(2) Suppose that $N$ is a proper submodule of $M$ and $M I \subseteq N$, where $I$ is an ideal of $R$. Since $M$ is comultiplication, $N=(0: M J)$ for some ideal $J$ of $R$. Then $M I \subseteq(0: M J)$ and so $M I J=0$. Since $\operatorname{ann}_{R}(M)$ is prime and $N \neq M$, we have $M I=0$. Thus $\operatorname{ann}_{R}(M)=\operatorname{ann}_{R}(M / N)$ and hence $M$ is a second module.

(3) For one direction, the proof is clear. For the other direction, assume that $N$ is both a secondary and a weakly second submodule of $M$. Let $I$ be an ideal of $R$ such that $N I \neq 0$ and $N I \neq N$. Since $N$ is secondary, there exists $n \geq 2$ such that $N I^{n}=0$. On the other hand, since $N$ is weakly second, we conclude that $N I=0$, a contradiction.

(4) Let $\left\{N_{i}\right\}_{i \in I}$ be a collection of weakly second submodules of $M$ and $N=\sum_{i \in I} N_{i}$. Clearly $N \neq 0$. Since for any $i \in I, N_{i}$ is weakly second, $\operatorname{ann}_{R}\left(N_{i}\right)$ is a prime ideal of $R$. Also $\operatorname{ann}_{R}(N)=\operatorname{ann}_{R}\left(\sum_{i \in I} N_{i}\right)=\bigcap_{i \in I} \operatorname{ann}_{R}\left(N_{i}\right)$ and since any two prime ideals of $R$ are comparable, $\operatorname{ann}_{R}(N)$ is a prime ideal of $R$. To complete the proof, it is enough to show that $\left(L:_{R} N\right)$ is a prime ideal of $R$, where $L$ is a submodule of $M$ such that $N \nsubseteq L$. Assume for two ideals $A$ and $B$ of $R, N A B \subseteq L$ such that $N A \nsubseteq L$ and $N B \nsubseteq L$. Then there exist $i, j \in I$ such that $N_{i} A \nsubseteq L$ and $N_{j} B \nsubseteq L$. This implies that

$$
A \nsubseteq \operatorname{ann}_{R}\left(\frac{N_{i}}{L \cap N_{i}}\right) \quad \text { and } \quad B \nsubseteq \operatorname{ann}_{R}\left(\frac{N_{j}}{L \cap N_{j}}\right) .
$$


Now since $N_{i}$ and $N_{j}$ are weakly second submodules of $M, \operatorname{ann}_{R}\left(\frac{N_{i}}{L \cap N_{i}}\right)$ and $\operatorname{ann}_{R}\left(\frac{N_{j}}{L \cap N_{j}}\right)$ are prime and by the assumption, these are comparable. Without loss of generality, we may assume that

$$
\operatorname{ann}_{R}\left(\frac{N_{i}}{L \cap N_{i}}\right) \subseteq \operatorname{ann}_{R}\left(\frac{N_{j}}{L \cap N_{j}}\right) .
$$

On the other hand, $N A B \subseteq L$ implies that $N_{i} A B \subseteq L$ and $N_{j} A B \subseteq L$. Thus $N_{i} A B \subseteq$ $L \cap N_{i}$ and $N_{j} A B \subseteq L \cap N_{j}$ and so $A B \subseteq \operatorname{ann}_{R}\left(\frac{N_{i}}{L \cap N_{i}}\right)$ and $A B \subseteq \operatorname{ann}_{R}\left(\frac{N_{j}}{L \cap N_{j}}\right)$. Since $\operatorname{ann}_{R}\left(\frac{N_{i}}{L \cap N_{i}}\right)$ and $\operatorname{ann}_{R}\left(\frac{N_{j}}{L \cap N_{j}}\right)$ are prime, by $(*)$, we have $A \subseteq \operatorname{ann}_{R}\left(\frac{N_{j}}{L \cap N_{j}}\right)$ and $B \subseteq$ $\operatorname{ann}_{R}\left(\frac{N_{i}}{L \cap N_{i}}\right)$. Now by $(* *), B \subseteq \operatorname{ann}_{R}\left(\frac{N_{j}}{L \cap N_{j}}\right)$, a contradiction.

Since every submodule of a comultiplication module is comultiplication and every quotient module of a multiplication module is also multiplication, the following result is immediate.

3.2. Corollary. Let $R$ be a ring and $M$ be a nonzero right $R$-module. Then:

(1) If $M$ is multiplication and $N$ is a weakly prime submodule of $M$, then $N$ is prime;

(2) If $M$ is comultiplication and $N$ is a weakly second submodule of $M$, then $N$ is second.

The following result shows that for an $R$-module $M$, if $R$ has the ascending chain condition (briefly, ACC) on two-sided ideals, then there exists a factor module of $M$ such that to be second.

3.3. Proposition. Let $R$ be a ring and $M$ be a nonzero right $R$-module. If ann $\left(M / N_{0}\right)$ is a maximal member in the family $\left\{a n n_{R}(M / N)\right\}$ where $N$ ranges over all proper submodules of $M$, then $M / N_{0}$ is a second $R$-module.

Proof. For any proper submodule $K / N_{0}$ of $M / N_{0}, \operatorname{ann}_{R}\left(M / N_{0}\right) \subseteq \operatorname{ann}_{R}(M / K)=$ $\operatorname{ann}_{R}\left(\frac{M / N_{0}}{K / N_{0}}\right)$ and the maximality of $\operatorname{ann}_{R}\left(M / N_{0}\right)$, implies that $\operatorname{ann}_{R}\left(M / N_{0}\right)=\operatorname{ann}_{R}\left(\frac{M / N_{0}}{K / N_{0}}\right)$. Thus $M / N_{0}$ is second $R$-module.

3.4. Proposition. Let $R$ be a ring and $M$ be a nonzero right $R$-module. If there exists a proper submodule $N$ of $M$ such that $M / N$ is a weakly second module, then ann $n_{R}(M / N)$ is a maximal member in the collection of ideals $I$ of $R$ such that $M I J+N \neq M J+N$ for every ideal $J$ of $R$ with $J \nsubseteq$ ann $_{R}(M / N)$.

Proof. Let $P=\operatorname{ann}_{R}(M / N)$ and $J$ be an ideal of $R$ such that $J \nsubseteq P$. Then clearly $M P J+N \neq M J+N$. Suppose that $A$ is an ideal of $R$ such that $P \subset A$. Then $A \nsubseteq P$ and since $M / N$ is weakly second, $(M / N) A^{2}=(M / N) A$. Thus $M A^{2}+N=M A+N$ and it follows that $P$ is a maximal member in the stated collection.

It was seen that a right $R$-module $M$ is weakly second if and only if $M I J=M I$ or $M I J=M J$ for every two ideals $I, J$ of $R$. Here, we improve this fact to modules over Noetherian rings.

3.5. Theorem. Let $R$ be a ring whose two-sided ideals satisfy ACC. Then a nonzero $R$-module $M$ is weakly second if and only if for every two prime ideals $P$ and $Q$ of $R$, $M P Q=M P$ or $M P Q=M Q$.

Proof. For one direction, the proof is clear. For the other direction assume that for every two prime ideals $P$ and $Q$ of $R, M P Q=M P$ or $M P Q=M Q$. First we show that for prime ideals $P_{1}, \cdots, P_{n}$ of $R, M P_{1} \cdots P_{n}=M P_{j}$ for some $1 \leq j \leq n$. We proceed by induction on $n$, the case $n=2$ being covered by hypothesis. For $n=3$, $M P_{1} P_{2} P_{3}=\left(M P_{1} P_{2}\right) P_{3}$ and by hypothesis, $M P_{1} P_{2}=M P_{1}$ or $M P_{1} P_{2}=M P_{2}$. Thus we have $M P_{1} P_{2} P_{3}=\left(M P_{1} P_{2}\right) P_{3}=\left(M P_{1}\right) P_{3}=M P_{1} P_{3}$ or $M P_{1} P_{2} P_{3}=\left(M P_{1} P_{2}\right) P_{3}=$ 
$\left(M P_{2}\right) P_{3}=M P_{2} P_{3}$. This shows that we are reduced to the case $n=2$. Again by hypothesis, $M P_{1} P_{3}=M P_{1}$ or $M P_{1} P_{3}=M P_{3}$ and $M P_{2} P_{3}=M P_{2}$ or $M P_{2} P_{3}=M P_{3}$. Therefore $M P_{1} P_{2} P_{3}=M P_{i}$ for some $1 \leq i \leq 3$. Now by the induction hypothesis, $M P_{1} \cdots P_{n}=\left(M P_{1} \cdots P_{n-1}\right) P_{n}=M P_{i} P_{n}$ for some $1 \leq i \leq n-1$. Also by the case $n=2$, we have $M P_{1} \cdots P_{n}=M P_{i} P_{n}=M P_{j}$ for some $1 \leq j \leq n$ and the claim is proved. Now let $I$ and $J$ be two ideals of $R$. Since $R$ has ACC on two-sided ideals, by [14, Lemma $1]$, there exist two integers $n, m$ and prime ideals $Q_{i}(1 \leq i \leq n)$ and $P_{j}(1 \leq j \leq m)$, such that

$$
Q_{1} \cdots Q_{n} \subseteq I \subseteq Q_{1} \cap \cdots \cap Q_{n}
$$

and

$$
P_{1} \cdots P_{m} \subseteq J \subseteq P_{1} \cap \cdots \cap P_{m}
$$

Thus

$$
Q_{1} \cdots Q_{n} P_{1} \cdots P_{m} \subseteq I J \subseteq\left(Q_{1} \cap \cdots \cap Q_{n}\right)\left(P_{1} \cap \cdots \cap P_{m}\right)
$$

and so

$$
M Q_{1} \cdots Q_{n} P_{1} \cdots P_{m} \subseteq M I J \subseteq M\left(Q_{1} \cap \cdots \cap Q_{n}\right)\left(P_{1} \cap \cdots \cap P_{m}\right) .
$$

By the first part of the proof, without loss of generality, we may assume that $M Q_{1} \cdots Q_{n} P_{1} \cdots P_{m}=M Q_{s}$ for some $1 \leq s \leq n$. Then we have $M Q_{s}=M Q_{1} \cdots Q_{n} P_{1} \cdots P_{m} \subseteq M I J \subseteq M\left(Q_{1} \cap \cdots \cap Q_{n}\right)\left(P_{1} \cap \cdots \cap P_{m}\right) \subseteq M Q_{s}$ and so $M I J=M Q_{s}$. On the other hand, $I \subseteq Q_{1} \cap \cdots \cap Q_{n}$ implies that $M I \subseteq M Q_{s}$ and hence $M I J=M I$.

Following [1, p. 232, ex. 11], a submodule $N$ of a right $R$-module $M$ is said to be pure (in $\mathrm{M}$ ) provided $N \cap M I=N I$ for every left ideal $I$ of $R$.

3.6. Proposition. Let $R$ be a ring and $M$ be a weakly second $R$-module. Then every pure submodule of $M$ is weakly second.

Proof. Let $N$ be a nonzero pure submodule of $M$ and $I, J$ be two ideals of $R$. If $M I J=M I$, then $N I J=N \cap M I J=N \cap M I=N I$. Similarly, $M I J=M J$ implies that $N I J=N J$. Now by Theorem 2.3 , the proof is complete.

A right $R$-module $X$ is called properly divisible if for every proper submodule $Y$ of $X$ and every regular element $c$ of $R, Y=Y c$.

3.7. Proposition. Let $R$ be a prime right Goldie ring. Then every nonzero properly divisible $R$-module is a weakly second module.

Proof. Let $X$ be a nonzero properly divisible $R$-module and $I, J$ be two ideals of $R$. If $X I=X$, then $X I J=X J$. Thus suppose that $X I \subset X$ and let $A=\operatorname{ann}_{R}(X I)$. If $A \neq 0$, then it is an essential ideal of $R_{R}$ (since $R$ is prime) and by the Goldie' $s$ Theorem [13, Theorem 11.13], $A$ contains a regular element $c$ of $R$. Since $X$ is properly divisible, $X I=X I c=0$ and so $X I J=X I$. Now assume that $A=0$ and $d$ is a regular element of $J$. Then $X I=X I d \subseteq X I J$ and hence $X I=X I J$, as desired.

A right $R$-module $M$ is called a semisecond module, if for any ideal $I$ of $R, M I^{2}=M I$, i.e., $\operatorname{ann}_{R}(M / N)$ is a semiprime ideal of $R$, for any proper submodule $N$ of $M$. We conclude the paper with the following result. 
3.8. Proposition. Let $R$ be a ring, $M$ be a right $R$-module and $N$ be a semisecond submodule of $M$ such that $M / N$ is second. If for any ideal $I$ of $R, N I$ is a weakly prime submodule of $M$, then $M$ is a semisecond module.

Proof. Let $I$ be an ideal of $R$. Since $M / N$ is second, $(M / N) I=M / N$ or $(M / N) I=0$. If $(M / N) I=M / N$, then $M I+N=M$ and so $M I^{2}+N I=M I$. Since $N$ is semisecond, $N I=N I^{2}$ and hence $M I^{2}=M I$. Now suppose that $(M / N) I=0$. Then $M I+N=N$ implies that $M I^{2}+N I=M I^{2}+N I^{2}=N I$ and so $M I^{2}=N I$, because $N$ is semisecond. Since $N I$ is a weakly prime submodule, $M I \subseteq N I$ and we have $M I^{2}=M I$.

\section{References}

[1] Anderson, F. W. and Fuller, K. R. Rings and Categories of Modules, New York: SpringerVerlag 1974

[2] Annin, S. Attached primes over noncommutative rings, J. Pure Appl. Algebra 212, 510-521, 2008.

[3] Ansari-Toroghy, H. and Farshadifar, F. The dual notion of multiplication modules, Taiwanese Journal of Mathematics 11, 1189-1201, 2007.

[4] Ansari-Toroghy, H. and Farshadifar, F. The dual notion of some generalizations of prime submodules, Comm. Algebra 39, 2396-2416, 2011.

[5] Azizi, A. On prime and weakly prime submodules, Vietnam J. Math. 36, 315-325, 2008.

[6] Azizi, A. Weakly prime submodules and prime submodules, Glasgow Math. J. 48, 343-346, 2006.

[7] Barnard, A. Multiplication modules, Journal of Algebra 71, 174-178, 1981.

[8] Behboodi, M. On weakly prime radical of modules and semi-compatible modules, Acta Math. Hungar. 113, 243-254, 2006.

[9] Behboodi, M. and Koohi, H. Weakly prime modules, Vietnam J. Math. 32, 185-195, 2004.

[10] Ceken, S. Alkan, M. and Smith, P. F. Second modules over noncommutative rings, Comm. Algebra 41, 83-98, 2013.

[11] Dauns, J. Prime modules, J. Reine Angew Math. 298, 156-181, 1978.

[12] Lam, T. Y. A first course in noncommutative rings, New York: Springer-Verlag 1991.

[13] Lam, T. Y. Lectures on modules and rings, Graduate Texts in Math. New York HeidelbergBerlin: Springer-Verlag 1999.

[14] Smith, P. F. Injective modules and prime ideals, Comm. Algebra 9, 989-999, 1981.

[15] Yassemi, S. The dual notion of prime submodules, Arch. Math. Brno. 37, 273-278, 2001. 
